\title{
Factores que influyen en el rendimiento académico de residentes de Obstetricia y Ginecología de una universidad en Bogotá, Colombia
}

\section{Factors that influence the academic performance of residents of Obstetrics and Gynecology of a university in Bogotá, Colombia}

\author{
Mario Arturo González Mariño
}

1M.D., M.Sc., D.Sc. Universidad Nacional de Colombia - sede Bogotá, Facultad de Medicina. Bogotá D.C., Colombia; e-mail: marioar90@hotmail.com;

(Dhttps://orcid.org/0000-0003-2970-336X

Cómo citar: González Mariño, M.A. 2019. Factores que influyen en el rendimiento académico de residentes de Obstetricia y Ginecología de una universidad en Bogotá, Colombia. Rev. U.D.C.A Act. \& Div. Cient. 22(2):e1377. http://doi.org/10.31910/rudca.v22. n2.2019.1377

Artículo de acceso abierto publicado por Revista U.D.C.A Actualidad \& Divulgación Científica, bajo una licencia Creative Commons CC BY-NC 4.0

Recibido: Julio 20 de 2018

Aceptado: Noviembre 4 de 2019

Editado por: Diana Paola Gutiérrez

\section{RESUMEN}

La formación de postgrado en medicina, conocida como Residencia, es un período de alta exigencia para el médico en formación. El rendimiento académico durante este período puede ser afectado por algunos factores socioeconómicos, psicoafectivos y otros que dependen de la característica del programa de postgrado. Para evaluar la influencia de estos factores en los residentes de Obstetricia y Ginecología de una universidad, se realizó un estudio descriptivo transversal. Los factores con mayor influencia en el rendimiento académico fueron los de contar con una motivación constante y poseer estabilidad económica propia y familiar. En la evaluación de los factores psicoafectivos, aquellos con mayores respuestas positivas fueron las consideraciones de que la función primordial es aprender, la percepción de que los exámenes no evalúan realmente lo aprendido y el hecho de enfrentar en el hospital los problemas emocionales con calma. Los residentes afirmaron que nunca han sido víctimas de intimidación, acoso, ofensas o agresiones por parte de sus compañeros. En conclusión, varios factores influyen, de manera muy importante, en el rendimiento académico de este grupo de residentes, principalmente, la estabilidad económica propia y de otros miembros de la familia, motivación constante, estar en función de aprendizaje, manejo con calma de los problemas emocionales y la apreciación de que los exámenes no evalúan realmente lo aprendido. Estos factores deben ser tenidos en cuenta porque, además de afectar la formación del residente, pueden también interferir en la atención de los pacientes y su identificación puede promover programas de bienestar para estos profesionales.

Palabras clave: factores socioeconómicos; factores psicoafectivos; rendimiento académico

\section{ABSTRACT}

Postgraduate training in medicine, known as Residence, is a period of high demand for the medical practitioner. The academic performance of this professional can be affected by some socioeconomic, psychoaffective and other factors that depend on the characteristic of the 
postgraduate program. To evaluate the influence of these factors on residents of Obstetrics and Gynecology of an university, a crosssectional descriptive study was performed. The factors with the greatest influence on academic performance were those of having a constant motivation and have their own and family economic stability. In the evaluation of psycho-affective factors, the factors with the highest positive responses were the consideration that the primary function as a resident is to learn, the perception that exams do not really assess what they have learned and the fact of facing emotional problems in the hospital with calm. All residents said they have never been victims of constant intimidation, harassment, offenses or attacks by any of their partners. In conclusion, several factors have a very important influence on the academic performance of this group of residents, mainly their own economic stability and of the other family members, constant motivation, being in a learning function, calmly handling emotional problems and the appreciation that exams do not really assess what they have learned. These factors must be taken into account, because in addition to affecting the resident's training they can also interfere with patient care and identifying them can promote wellness programs for these professionals.

Keywords: socioeconomic factors; psycho-affective factors; academic performance

\section{INTRODUCCIÓN}

Según la Organización Panamericana de la Salud (OPS, 2011), se define como residencia médica al sistema educativo que tiene por objeto completar la formación de los médicos en alguna especialización reconocida por su país de origen, mediante el ejercicio de actos profesionales de complejidad y de responsabilidad progresivas, llevados adelante bajo supervisión de tutores en instituciones de servicios de salud y con un programa educativo aprobado para tales fines.

La formación de especialistas médicos es un proceso largo y complejo, cuyo objetivo primordial es garantizarle a la sociedad que sean profesionales idóneos para atender las necesidades de salud de la población (Jaramillo \& Martin, 2012).

En Colombia, los residentes son considerados como estudiantes (Reyes \& Ortiz, 2013). Hasta la promulgación de la ley 1917 de 12 julio de 2018 (reglamentada por el Ministerio de Salud y Protección Social mediante la Resolución 1872 de Julio 12 de 2019) (República de Colombia, 2018), no recibían una remuneración económica y pagaban (continuarán pagando) matrícula durante su período de formación. Además, se exige, por la gran mayoría de programas de postgrado dedicación exclusiva, lo que obliga a muchos residentes a buscar trabajos alternos, sin el consentimiento de su facultad, en detrimento de una adecuada formación (Bermúdez et al. 2006).

En este tiempo de alta exigencia académica, el residente puede ser afectado por factores socioeconómicos, psicoafectivos y otros que dependen de la característica del programa de postgrado, que pueden disminuir el rendimiento académico. La fatiga que ocasionan los turnos y el demandante trabajo del residente en áreas asistenciales debe ser tenida en cuenta, porque pueden generar efectos negativos en la atención y en el aprendizaje (Ministerios de Salud y Educación, 2017). En México, Manterola (2015) aplicó una encuesta en un hospital público de tercer nivel de atención, con el objetivo de determinar la influencia de varios factores en el desempeño académico de los médicos residentes de la especialidad de Ginecología y Obstetricia.

Con el propósito de conocer cómo afectan los factores propuestos por Manterola en el rendimiento académico de residentes de la misma especialidad en Colombia, se aplicó este instrumento, con algunas modificaciones.

\section{MATERIALES Y METODOS}

Se trata de un estudio descriptivo transversal de tipo encuesta, en el cual, se hizo una invitación a participar, de manera voluntaria y de forma anónima, a todos los residentes de la especialidad de Obstetricia y Ginecología de un programa de postgrado en Bogotá, Colombia. Se aplicó un formulario de preguntas sobre el año de residencia, edad, género, estado civil y número de personas dependientes. También, se usó una escala tipo Likert con valores de poco, más o menos y mucho sobre factores socioeconómicos, psicoafectivos y propios del programa y otro instrumento para marcar respuestas afirmativas o negativas sobre factores psicoafectivos. Los factores evaluados fueron tomados, con algunas modificaciones, de la encuesta publicada por Manterola (2015) (con permiso de su autor). Esta encuesta, se aplicó en noviembre de 2016. Para el análisis se usaron porcentajes, medidas de tendencia central y de dispersión.

\section{RESULTADOS Y DISCUSIÓN}

De un total de 29 residentes, se recibieron 23 encuestas (79,3\%). El promedio de edad de los residentes que respondieron la encuesta fue 27,73 años, con moda según el año de residencia de 26, 28 y 31 años, para los años uno a tres, respectivamente. La mayoría mujeres $(78,26 \%)$ y de estado civil soltero $(91,30 \%)$. Las características generales de los residentes participantes en la encuesta, se presentan en la tabla 1.

Factores que contribuyen a mejorar el rendimiento académico: La estabilidad económica propia y de otros miembros de la familia junto con tener una motivación constante, se califican, en todas las encuestas, como muy importantes en el rendimiento académico.

Otro factor considerado como muy importante, por más del 95\% de los participantes, fue el tener acceso gratuito a bases de datos y libros electrónicos. El siguiente factor, en frecuencia, fue el cumplimiento de las actividades académicas (91\%).

Tener buen hábito de sueño o sueño reparador fue calificado en $87 \%$ como muy importante en el rendimiento académico y, en orden descendiente, mantener buenas relaciones con los familiares y desarrollar un tipo de aprendizaje autodirigido (Tabla 2). 
Tabla 1. Características generales de los residentes.

\begin{tabular}{|c|c|c|c|c|c|c|}
\hline \multirow{3}{*}{$\begin{array}{c}\text { Residentes } \\
\text { (número de respuestas) } \\
\text { Primer año } \\
(9)\end{array}$} & \multirow{3}{*}{$\begin{array}{c}\text { Edad } \\
\text { Promedio } \\
\text { (rango) Años } \\
26,44 \\
(24-30)\end{array}$} & \multicolumn{2}{|c|}{ Género } & \multirow{2}{*}{\multicolumn{2}{|c|}{$\begin{array}{c}\text { Estado Civil } \\
(\%)\end{array}$}} & \multirow{3}{*}{$\begin{array}{c}\begin{array}{c}\text { Personas } \\
\text { dependientes }\end{array} \\
0 \\
(1 \text { no reporta }) \\
\end{array}$} \\
\hline & & $\mathrm{F}(\%)$ & $\mathrm{M}(\%)$ & & & \\
\hline & & $7(78)$ & $2(22)$ & $\begin{array}{l}\text { Soltero } \\
\text { Unión Libre }\end{array}$ & $\begin{array}{l}8(89) \\
1(11) \\
\end{array}$ & \\
\hline $\begin{array}{c}\text { Segundo año } \\
(8)\end{array}$ & $\begin{array}{c}28,37 \\
(26-35)\end{array}$ & $7(88)$ & $1(12)$ & Soltero & $8(100)$ & 0 \\
\hline $\begin{array}{c}\text { Tercer año } \\
(6)\end{array}$ & $\begin{array}{c}28,83 \\
(24-31)\end{array}$ & $4(67)$ & $2(33)$ & $\begin{array}{l}\text { Soltero } \\
\text { Unión Libre }\end{array}$ & $\begin{array}{l}5(83) \\
1(17)\end{array}$ & $1^{*}$ \\
\hline
\end{tabular}

* En un formulario se reporta una persona dependiente.

Tabla 2. Respuestas a factores que pueden afectar el rendimiento académico.

\begin{tabular}{|c|c|c|c|c|c|c|}
\hline \multirow{2}{*}{$\begin{array}{c}\text { Factores } \\
\text { Tener buen hábito de sueño o sueño reparador }\end{array}$} & \multicolumn{2}{|c|}{$\begin{array}{c}\text { Poco } \\
(\%)\end{array}$} & \multicolumn{2}{|c|}{$\begin{array}{l}\text { Más o menos } \\
(\%)\end{array}$} & \multicolumn{2}{|c|}{$\begin{array}{l}\text { Mucho } \\
(\%)\end{array}$} \\
\hline & 0 & (0) & 3 & (13) & 20 & (87) \\
\hline Desarrollar un tipo de aprendizaje autodirigido & 0 & $(0)$ & 5 & $(22)$ & 18 & $(78)$ \\
\hline Estabilidad económica propia y de otros miembros de la familia & 0 & $(0)$ & 0 & $(0)$ & 23 & $(100)$ \\
\hline Tener un tutor & 2 & $(9)$ & 12 & $(52)$ & 9 & $(39)$ \\
\hline Que se cumplan las actividades académicas & 0 & $(0)$ & 2 & $(9)$ & 21 & $(91)$ \\
\hline $\begin{array}{l}\text { Tener una motivación constante a pesar de la excesiva carga de } \\
\text { trabajo }\end{array}$ & 0 & $(0)$ & 0 & $(0)$ & 23 & $(100)$ \\
\hline Es importante el nivel socio económico al cual se pertenece & 10 & $(43)$ & 10 & $(43)$ & 3 & $(13)$ \\
\hline Tener una pareja sentimental estable & 5 & $(22)$ & 11 & $(48)$ & 7 & $(30)$ \\
\hline Trabajar en un entorno seguro y con los recursos suficientes & 1 & $(4)$ & 2 & $(9)$ & 20 & $(87)$ \\
\hline Tener acceso gratuito a bases de datos y libros electrónicos & 0 & $(0)$ & 1 & $(4)$ & 22 & $(96)$ \\
\hline Contar con recursos tecnológicos de última generación & 2 & $(9)$ & 10 & $(43)$ & 11 & $(48)$ \\
\hline Tener algún familiar médico & 20 & $(87)$ & 3 & $(13)$ & 0 & $(0)$ \\
\hline Ambiente de trabajo con disciplina estricta & 5 & $(22)$ & 16 & $(69)$ & 2 & $(9)$ \\
\hline Publicar artículos en revistas médicas o científicas & 6 & $(26)$ & 8 & $(35)$ & 9 & $(39)$ \\
\hline Evaluaciones académicas constantes & 6 & $(26)$ & 10 & $(43)$ & 7 & $(30)$ \\
\hline Buenas relaciones con los familiares & 0 & $(0)$ & 4 & $(17)$ & 19 & $(83)$ \\
\hline Medio de transporte propio & 8 & $(35)$ & 14 & $(61)$ & 1 & $(4)$ \\
\hline Competencia constante con los demás compañeros & 13 & $(56)$ & 8 & $(35)$ & 2 & $(9)$ \\
\hline Acceso gratuito a internet dentro del hospital & 1 & $(4)$ & 5 & $(22)$ & 17 & $(74)$ \\
\hline
\end{tabular}

Factores psicoafectivos que influyen el rendimiento académico: Los factores con mayores respuestas positivas fueron: "mi función primordial como residente es aprender", "los exámenes no evalúan realmente lo aprendido durante la residencia" y "en el hospital enfrento mis problemas emocionales con calma", cada uno con 91\% (Tabla 3). 
Tabla 3. Influencia de factores psicoafectivos en el rendimiento académico.

\begin{tabular}{|c|c|c|c|c|}
\hline \multicolumn{5}{|l|}{ FACTORES PSICOAFECTIVOS } \\
\hline & \multicolumn{2}{|c|}{ SI $(\%)$} & \multicolumn{2}{|c|}{ NO $(\%)$} \\
\hline Mi función primordial como residente es aprender & 21 & $(91)$ & 2 & $(9)$ \\
\hline Me afecta algún tipo de pelea con mis compañeros de residencia & 15 & $(65)$ & 8 & (35) \\
\hline Trabajo mejor con mis compañeros(as) del mismo sexo & 5 & $(22)$ & 18 & $(78)$ \\
\hline He sido víctima de maltrato, abuso, discriminación por parte de mis compañeros de residencia & 3 & (13) & 20 & $(87)$ \\
\hline Durante mi residencia he tenido algún conflicto que me ha hecho pensar en renunciar & 4 & $(17)$ & 19 & $(83)$ \\
\hline $\begin{array}{l}\text { Mi residente de mayor jerarquía debe resolver todas las situaciones conflictivas que se presenten entre } \\
\text { los residentes de menor jerarquía. }\end{array}$ & 2 & (9) & 21 & (91) \\
\hline Considero a mis residentes de mayor jerarquía verdaderos líderes en quienes puedo confiar & 13 & $(57)$ & 10 & $(43)$ \\
\hline $\begin{array}{l}\text { He cometido algún tipo de abuso, maltrato, hostigamiento, acoso o discriminación a algún residente de } \\
\text { menor o mayor jerarquía durante mi residencia }\end{array}$ & 4 & $(17)$ & 19) & (83) \\
\hline $\begin{array}{l}\text { Mi cansancio ha sido tan extremo que he maltratado a las pacientes sin querer hacerlo intencional- } \\
\text { mente }\end{array}$ & 4 & $(17)$ & 19 & (83) \\
\hline Me he sentido triste, agobiado(a) o sin ganas de trabajar durante algún momento de mi residencia & 17 & $(74)$ & 6 & $(26)$ \\
\hline $\begin{array}{l}\text { Tuve períodos de mucha angustia, inseguridad, temor o estrés al momento de tomar una decisión diag- } \\
\text { nóstica o terapéutica de alguna paciente }\end{array}$ & 15 & $(65)$ & 8 & (35) \\
\hline En mi tiempo libre prefiero descansar, distraerme, salir con mis amigos o dormir en lugar de estudiar & 17 & (74) & 6 & (26) \\
\hline El tipo de trabajo que desempeño en la residencia es muy rígido y repetitivo & 8 & $(35)$ & 15 & $(65)$ \\
\hline Durante los turnos no se deberían programar clases & 10 & $(43)$ & 13 & $(57)$ \\
\hline $\begin{array}{l}\text { Constantemente soy víctima de intimidación, acoso, ofensas o agresiones por parte de alguno de mis } \\
\text { compañeros residentes }\end{array}$ & 0 & $(0)$ & 23) & $(100)$ \\
\hline En algún momento mi trabajo he sido menospreciado a pesar de haberlo hecho correctamente & 13 & $(57)$ & 10 & (43) \\
\hline Durante las revistas con pacientes he sido muy criticado injustamente frente a mis compañeros & 5 & $(22)$ & 18 & (78) \\
\hline Mi autoestima ha sido dañada en algún momento durante mi residencia & 9 & & 14 & (61) \\
\hline Los especialistas tratan mucho mejor a mis compañeros(as) del sexo opuesto & 5 & $(22)$ & 18 & $(78)$ \\
\hline Mi estado de ánimo empeoró desde que entré a la residencia & 8 & (35) & 15 & $(65)$ \\
\hline Algunas veces no me siento capaz de realizar mis actividades como siempre las he hecho & 14 & $(61)$ & 9 & $(39)$ \\
\hline Las largas jornadas de trabajo y los turnos limitan mis actividades académicas & 20 & $(87)$ & 3 & (13) \\
\hline He estado muy triste y deprimido(a) en algún momento durante mi residencia & 15 & $(65)$ & 8 & (35) \\
\hline $\begin{array}{l}\text { La excesiva carga de trabajo y el estrés me han inducido en algún momento a consumir bebidas al- } \\
\text { cohólicas de manera frecuente }\end{array}$ & 1 & (4) & 22 & (96) \\
\hline Fumar me relaja siempre que estoy bajo mucho estrés & 2 & $(9)$ & 21 & (91) \\
\hline Los exámenes no evalúan realmente lo aprendido durante la residencia & 21 & (91) & 2 & $(9)$ \\
\hline A veces no me gusta enseñar a otros residentes que no me caen bien & 2 & $(9)$ & 21 & (91) \\
\hline En el hospital enfrento mis problemas emocionales con calma & 21 & $(91)$ & 2 & $(9)$ \\
\hline Algunas veces creo que me he vuelto más insensible con las personas desde que entré a la residencia & 9 & $(39)$ & 14 & $(61)$ \\
\hline A veces me siento frustrado en mi trabajo como residente & 16 & (70) & 7 & (30) \\
\hline
\end{tabular}

El factor que concitó el mayor número de respuestas negativas $(100 \%)$ fue contra la afirmación de ser constantemente víctima de intimidación, acoso, ofensas o agresiones por parte de alguno de los compañeros residentes.
La evaluación de otros factores encuentra que cerca del $87 \%$ de los residentes evaluados, afirma que las largas jornadas de trabajo y los turnos limitan las actividades académicas y, el 91\% de ellos, considera que los exámenes no evalúan realmente lo aprendido durante la residencia. 
La formación de especialistas médicos implica una gran inversión de recursos por las entidades formadoras (universidades, instituciones de salud), así como grandes esfuerzos, dinero y dedicación de tiempo completo por parte de los residentes (Jaramillo \& Martin, 2012).

Durante el tiempo de residencia, se pueden presentar factores que afecten el desempeño académico. En este grupo, a pesar de ser solteros y no tener personas dependientes, en su gran mayoría, todos tienen una gran preocupación con la parte económica propia y la de su familia, solo comparable en su impacto sobre el rendimiento académico con la necesidad de motivación constante. La preocupación económica no fue tan importante en el estudio realizado en México (55\% con calificación de muy importante) (Manterola, 2015); sin embargo, la situación es diferente, ya que allí el residente sí recibe un pago durante su formación (Reyes \& Ortiz, 2013), en tanto que, en Colombia, el médico para convertirse en especialista debe todavía pagar los costos, a pesar de estar ya graduado y desempeñar actividades asistenciales, propias de su nivel de entrenamiento (Bermúdez et al. 2006).

El acceso gratuito a bases de datos y libros electrónicos fue evaluado con más del 95\% de respuestas como factor muy asociado con el rendimiento académico; en el caso específico de estos residentes, es provisto por la universidad.

Tener buen hábito de sueño o sueño reparador también fue considerado muy importante $(80 \%)$, en el estudio realizado en un hospital general público de tercer nivel de México (Manterola, 2015).

En nuestro estudio, desarrollar un tipo de aprendizaje autodirigido fue calificado en mayor proporción como muy importante, contrario a lo reportado en el estudio de México, donde el 50\% lo consideró muy importante (Manterola, 2015).

En la evaluación de factores psicoafectivos, en este programa de residencia a diferencia de otros, no se reportó la intimidación en el lugar de trabajo, "bullying" (definido como comportamiento persistente hacia un individuo consistente en ser intimidante, degradante, ofensivo o malicioso y que socava la confianza y autoestima del receptor) (Paice \& Smith, 2009). Entre los residentes de un hospital pediátrico de México, se reportó ser víctima de bullying, en el $32 \%$, de manera espontánea, pero al interrogar sobre el tema subió al 82,4\% (Sepúlveda et al. 2017). Un estudio realizado con 421 residentes de tres hospitales de Buenos Aires reveló que $90 \%$ indicaron haber recibido algún tipo de maltrato (Mejía et al. 2005). En Chile, $71 \%$ de los encuestados refirieron haber experimentado alguna conducta de acoso durante el último año y casi $9 \%$ sufrieron acoso de manera frecuente. Las conductas más usuales incluyeron los intentos por menospreciar y menoscabar su trabajo y las críticas injustificadas al supervisar su actividad (Bastías et al. 2011). En un estudio longitudinal en el que participaron 260 residentes de las especialidades médicas de cirugía, medicina interna, ginecología y obstetricia y pediatría de tres hospitales generales de la Ciudad de México, se encontró que 256 (98,5\%) residentes manifestaron haber sufrido, por lo menos, una conducta de acoso durante los últimos seis meses (Ortiz et al. 2014).
A pesar del resultado de que las largas jornadas de trabajo y los turnos limitan las actividades académicas, en este grupo de residentes se está aplicando la normatividad sobre el número de horas de servicio, contempladas en el decreto 2376 de 2010 del Ministerio de la Protección Social de Colombia, que regula la relación docencia servicio para los programas de formación de talento humano del área de la salud, mediante el cual, "los turnos de las prácticas formativas de los estudiantes se fijarán atendiendo las normas, principios y estándares de calidad en la prestación del servicio de salud y de bienestar de los estudiantes y docentes. En cualquier caso, los turnos serán de máximo 12 horas, con descansos que garanticen al estudiante su recuperación física y mental y no podrán superar 66 horas por semana" (Ministerio de la Protección Social, 2010).

Si bien la encuesta aplicada no es específica para detectar depresión y "burnout", el predominio de respuestas positivas ante enunciados de haber estado muy triste y deprimido(a) en algún momento durante la residencia (65\%), algunas veces no sentirse capaz de realizar las actividades "como siempre las he hecho" $(61 \%)$, a veces sentirse frustrado en el trabajo como residente $(69 \%)$ y las respuestas afirmativas minoritarias en el enunciado de creerse más insensible con las personas desde que entró a la residencia $(39 \%)$, pueden corresponder a síntomas de éstas dos patologías; en cuanto a la depresión, la frecuencia es menor que en el estudio de Manterola (2015) $(80 \%)$ y es mayor que en otros reportes, que la sitúan en un rango de 7 y $56 \%$ (Fahrenkopf et al. 2008). Estas respuestas también se pueden asociar con el síndrome de "burnout", o síndrome de agotamiento profesional, que es un problema que se manifiesta en el personal de salud que se encuentra en contacto con pacientes. Es el resultado de la interacción entre factores personales comunes a muchos profesionales y específicos de la organización hospitalaria, en donde se desempeñan. Se caracteriza por tres manifestaciones: agotamiento emocional, despersonalización o cinismo y pérdida del logro profesional (Maslach \& Leiter, 1997). Esta enfermedad, reconocida por el Ministerio de Trabajo de Colombia, por el Decreto 1477 de 2014, como enfermedad laboral, se presenta con frecuencia en residentes. Existen evidencias que demuestran que la residencia puede causar un grado considerable de "burnout", debido a los problemas relacionados con el cuidado de los pacientes, incluyendo dilemas y errores diagnósticos, error en prescripción de medicamentos, tratamientos complejos, toma de decisiones, atención subóptima de pacientes (Shanafelt et al. 2002) y poca satisfacción del paciente con el tratamiento (Haas et al. 2000; Passalacqua \& Segrin, 2012).

En España, se reporta una prevalencia del 58\% de "burnout" en médicos residentes de Ginecología y Obstetricia, encontrando, como factores predisponentes, la sobrecarga de trabajo sin supervisión y ser soltero (Castelo-Branco et al. 2007). En diferentes estudios, se registran reportes de "burnout" entre los residentes entre 41 y 76\% (Thomas, 2004).

Las evaluaciones sobre el consumo de bebidas alcohólicas de manera frecuente y fumar arrojaron resultados muy bajos, diferente a otros estudios que han evaluado estos factores, con reportes por encima del 35\% (Manterola, 2015). 
Desde hace varios años existe la tendencia a cambiar los modelos tradicionales de enseñanza en medicina por otros aún sin demostración clara de ser mejores (Frank et al. 2010); sin embargo, es interesante el hallazgo de que este grupo considera que los exámenes no evalúan realmente lo aprendido durante la residencia.

Conocer la apreciación que de estos factores tienen los residentes es importante para comprender cómo se desarrolla la formación de los médicos durante este período de postgrado, de alta exigencia académica y laboral, en preparación para el ejercicio de la especialidad. A partir de esta información, se pueden generar estudios que permitan profundizar aspectos específicos para valorar el rendimiento y los factores psicoafectivos, presentes en estas personas.

La formación como especialista en Colombia ha sido tradicionalmente una etapa difícil para el médico graduado, no solamente por el alto número de horas dedicadas a los programas, sino también por el impacto económico, que este periodo de formación representa; afortunadamente, estas dos barreras han tenido modificaciones reglamentarias para mejorar sus condiciones. El aspecto económico de la falta de pago hace que este grupo estudiado sea diferente al que se evalúa en otros países, pero comparte la exigencia de aprender un gran volumen de conocimientos y de adquirir destrezas en muchos procedimientos. Se asume que este tiempo de formación es como tal de apoyo docente y no asistencial, pero con frecuencia termina en lo segundo, generando angustia e inconformidad en el residente, quien se ve agobiado por la incorporación al currículo de nuevos temas y tecnologías, que debe aprender en menos tiempo. Un compromiso de los responsables de estos programas debe ser el mejorar la calidad de vida del futuro especialista y brindar mejores condiciones de bienestar y de acompañamiento.

Conflicto de intereses: El manuscrito fue preparado y revisado por el autor. Declaro que no existe conflicto de intereses que ponga en riesgo la validez de los resultados presentados.

Consideraciones éticas: Se trata de un estudio, en el cual, se hizo una invitación a participar, de manera voluntaria y de forma anónima, mediante el diligenciamiento de un formulario. Adoptando la norma correspondiente a la investigación en salud en Colombia, se considera una investigación sin riesgo: Resolución No. 008430 de 1993, por la cual, se establecen las normas científicas, técnicas y administrativas para la investigación en salud en Colombia, artículo 11, a) Investigación sin riesgo: "Son estudios que emplean técnicas y métodos de investigación documental retrospectivos y aquellos en los que no se realiza ninguna intervención o modificación intencionada de las variables biológicas, fisiológicas, sicológicas o sociales de los individuos que participan en el estudio, entre los que se consideran: revisión de historias clínicas, entrevistas, cuestionarios y otros en los que no se le identifique ni se traten aspectos sensitivos de su conducta".

\section{REFERENCIAS}

1. BASTÍAS, N.; FASCE, E.; ORTIZ, L.; PÉREZ, C.; SCHAUFELE, P. 2011. Bullying y acoso en la formación médica de posgrado. Rev. Educ. Cienc. Salud. 8:45-51.

2. BERMÚDEZ, CH.E.; MONROY, A.; TORREGROSA, L.; HENAO, F. 2006. Estado actual de la formación de residentes de cirugía general en Colombia. Rev. Col. Cir. 21(4):225-239.

3. CASTELO-BRANCO, C.; FIGUERAS, F.; EIXARCH, E.; QUEREDA, F.; CANCELO, M.J.; GONZÁLEZ, S.; BALASCH, J. 2007. Stress symptoms and burnout in obstetrics and gynecology residents. BJOG. 114(1):94-98. https://doi.org/10.1111/j.1471-0528.2006.01155.x

4. FRANK, J.R.; SNELL, L.S.; CATE, O.T.; HOLMBOE, E.S.; CARRACCIO, C.; SWING, S.R.; HARRIS, P.; GLASGOW, N.J.; CAMPBELL, C.; DATH, D.; HARDEN, R.M.; IOBST, W.; LONG, D.M; MUNGROO, R.; RICHARDSON, D.L.; SHERBINO, J.; SILVER, I.; TABER, S.; TALBOT, M.; HARRIS, K.A. 2010. Competency-based medical education: theory to practice. Medical Teacher. 32:638-645. https://doi.org/10.3109/0142159X.2010.501190

5. FAHRENKOPF, A.M.; SECTISH, T.C.; BARGER, L.K.; SHAREK, P.J.; LEWIN, D.; CHIANG, V.W.; EDWARDS, S.; WIEDERMANN, B.L.; LANDRIGAN C.P. 2008. Rates of medication errors among depressed and burnout residents: prospective cohort study. BMJ. 336(7642):488489. https://doi.org/10.1136/bmj.39469.763218

6. HAAS, J.S.; COOK, E.F.; PUOPOLO, A.L.; BURSTIN, H.R.; CLEARY, P.D.; BRENNAN, T.A. 2000. Is the professional satisfaction of general internists associated with patient satisfaction? J. Gral Internal. Medicine. 15(2):122-128. https://doi.org/10.1046/j.1525-1497.2000.02219.x

7. JARAMILLO, L.E.; MARTIN, E. 2012. Evaluación integral de residentes de psiquiatría: un complemento del proceso de admisión al programa. Rev. Col. Psiquiat. 41(Suplemento):104S-112S. https://doi.org/10.1016/ S0034-7450(14)60183-9

8. MANTEROLA, D. 2015. Factores socioeconómicos y psicoafectivos y su influencia en el rendimiento académico de los residentes de Ginecología y Obstetricia. Ginecol Obstet. Mex. 83:139-147.

9. MASLACH, C.; LEITER, M. 1997. The truth about burnout. San Francisco: Jossey-Bass Publishing. 182p. 
10. MEJÍA, R.; DIEGO, A.; ALEMÁN, M.; MALIANDI, M.R.; LASALA, F. 2005. Percepción de maltrato durante la capacitación de médicos residentes. Medicina (Argentina). 65:366-368. https://doi.org/10.1016/j.riem.2017.04.004

11. MINISTERIO DE SALUD Y PROTECCIÓN SOCIAL DE COLOMBIA. 2019. Resolución 1872, "Por la cual se reglamenta la Ley 1917 de 2018 que creó el Sistema de Residencias Médicas en Colombia, su mecanismo de financiación y se dictan otras disposiciones". Disponible desde Internet en: https://www.minsalud.gov.co/ Normatividad_Nuevo/Resoluci $\%$ C3 $\%$ B3n $\% 20$ No. $\% 20$ 1872\%20de\%202019.pdf [con acceso el 27/08/2019].

12. MINISTERIO DE SALUD Y PROTECCIÓN SOCIAL DE COLOMBIA Y MINISTERIO DE EDUCACION NACIONAL. 2017. Recomendaciones para la transformación de la educación médica en Colombia. Disponible desde Internet en: https://www.minsalud.gov. $\mathrm{co} /$ sites/rid/Lists/BibliotecaDigital/RIDE/VS/MET/ recomendaciones-comision-para-la-transformacion.pdf [con acceso el 27/08/2019].

13. MINISTERIO DE LA PROTECCIÓN SOCIAL DE COLOMBIA. 2010. Decreto número 2376, "Por medio del cual se regula la relación docencia - servicio para los programas de formación de talento humano del área de la salud". Disponible desde Internet en: https://www. minsalud.gov.co/Normatividad_Nuevo/DECRETO $\% 20$ 2376\%20DE\%202010.pdf [con acceso el 7/08/2017].

14. MINISTERIO DEL TRABAJO DE COLOMBIA. 2014. Decreto número 1477, "Por el cual se expide la Tabla de Enfermedades Laborales". Disponible desde Internet en: http://www.bogotaturismo.gov.co/sites/intranet. bogotaturismo.gov.co/files/decreto_1477_del_5_de_ agosto_de_2014\%5B1\%5D.pdf [con acceso el 7/08/2017].

15. ORGANIZACIÓN PANAMERICANA DE LA SALUD, OPS. Residencias Médicas en América Latina. 2011. Serie: La renovación de la atención primaria en Salud en las Américas No 5. OPS. Washington, D.C.: OPS.
16. ORTIZ, S.; JAIMES, A.L.; TAFOYA, S.A.; MUJICA, M.L.; OLMEDO, V.H.; CARRASCO, J.A. 2014. Experiencias de maltrato y hostigamiento en médicos residentes. Cir Cir. 82:290-301.

17. PAICE, E.; SMITH, D. 2009. Bullying of trainee doctors is a patient safety issue. The Clinical Teacher. 6(1):13-17. https://doi.org/10.1111/j.1743-498X.2008.00251.x

18. PASSALACQUA, S.A.; SEGRIN, C. 2012. The effect of resident physician stress, burnout, and empathy on patientcentered communication during the longcall shift. Health communication. 27(5):449-456. https://doi.org/10.1080/ 10410236.2011.606527

19. REPÚBLICA DE COLOMBIA. 2018. Ley No. 1917, “Por medio de la cual se reglamenta el sistema de Residencias Médicas en Colombia, su mecanismo de financiación y se dictan otras disposiciones".

20. REYES, G.; ORTIZ, L.C. 2013. Sistema de Residencias Médicas en Colombia: Marco conceptual para una propuesta de regulación, Ministerio de Salud y Protección Social Bogotá D.C.

21. SEPÚlVEDA-VILDÓSOLA, A.C.; MOTA-NOVA, A.R.; FAJARDO-DOLCI, G.E.; REYES-LAGUNES, L.I. 2017. Workplace bullying during specialty training in a pediatric hospital in Mexico: a little-noticed phenomenon. Rev Med Inst Mex Seguro Soc.55 (Suppl 1): S92-S101.

22. SHANAFELT, T.D.; BRADLEY, K.A.; WIPF, J.E.; BACK, A.L. 2002. Burnout and self-reported patient care in an internal medicine residency program. Annals of internal medicine. 136(5):358-367. https://doi.org/10.7326/0003-4819-1365-200203050-00008

23. THOMAS, N.K. 2004. Resident burnout. JAMA. 292:28802889. https://doi.org/10.1001/jama.292.23.2880 